Pacific Journal of Mathematics

SETS OF INTEGERS CLOSED UNDER AFFINE
OPERATORS-THE CLOSURE OF FINITE SETS 


\title{
SETS OF INTEGERS CLOSED UNDER AFFINE OPERATORS-THE CLOSURE OF FINITE SETS
}

\author{
D. G. Hoffman AND D. A. Klarner
}

We continue investigation begun in 1974 of sets of integers closed under operators of the form $\left(x_{1}, \cdots, x_{r}\right) \rightarrow$ $m_{1} x_{1}+\cdots+m_{r} x_{r}+c$, where $m_{1}, \cdots, m_{r}$ are integers with $\operatorname{gcd}\left(m_{1}, \cdots, m_{r}\right)=1$. Our main goal here is to prove the following.

Theorem 12. Let $r, m_{1}, \cdots, m_{r}$ be positive integers, let $T$ be a set of integers, let $c$ be an integer such that $\left(m_{1}+\cdots+m_{r}-1\right) t+c$ is positive for each $t \in T$. If $\operatorname{gcd}\left(m_{1}, \cdots, m_{r}\right)=1$, and if $T$ is closed under the operator $\left(x_{1}, \cdots, x_{r}\right)\left(x_{1}, \cdots, x_{r}\right) m_{1} x_{1}+\cdots+m_{r} x_{r}+c$, then the following two statements are equivalent: sions.

(1) $T$ is a finite union of infinite arithmetic progres-

(2) $T=\left\langle m_{1} x_{1}+\cdots+m_{r} x_{r}+c \mid A\right\rangle$ for some finite set $A$, where $\left\langle m_{1} x_{1}+\cdots+m_{r} x_{r}+c \mid A\right\rangle$ denotes the "smallest" set containing $A$, and closed under the operator $\left(x_{1}, \cdots, x_{r}\right) \rightarrow$ $m_{1} x_{1}+\cdots+m_{r} x_{r}+c$.

In fact, (1) and (2) are true under more general conditions; these extensions are made in [1].

Notation. We denote by $\boldsymbol{Z}, \boldsymbol{N}$, and $\boldsymbol{P}$ the set of integers, the set of nonnegative integers, and the set of positive integes, respectively. If $A, B \subseteq Z$, and $c \in Z$, define $A+c=\{a+c \mid a \in A\}, c A=\{c a \mid a \in A\}$, and $A+B=\{a+b \mid a \in A, b \in B\}$. If $a, b \in Z$, define $[a, b]=\{c \in Z \mid a \leqq$ $c \leqq b\}$. If $A$ and $B$ are sets, we write $A \subseteq B$ when $A \backslash B$ is finite, and $A \doteq B$ when $A \sqsubseteq B \sqsubseteq A$.

We begin by discussing sets satisfying (1).

A subset $A \subseteq Z$ is a periodic set if there exists a finite set $I$, and for each $i \in I$, an integer $a_{i}$, and a positive integer $d_{i}$, with $A=$ $\mathrm{U}_{i \in I}\left(a_{i}+d_{i} N\right)$.

It is easy to see that $A$ is periodic iff $A$ is bounded below, and

$$
A+d \subseteq A \text { for some } d \in P .
$$

For the proofs of the elementary properties of periodic sets we shall use, see [3], for though the "per-set" defined there is slightly different from the one defined here, the difference is not essential.

A $d \in \boldsymbol{P}$ satisfying (3) is called a period of $A$. However, a $d \in \boldsymbol{P}$ 
is an eventual period of a subset $A \subseteq Z$ if $A$ is a periodic set, and $A+d \risingdotseq A$.

We state without proof the following elementary properties of periodic sets and their eventual periods.

\section{LEMMA 1.}

(i) If $A$ is a periodic set, then for some $d \in \boldsymbol{P}, d \boldsymbol{P}$ is the set of eventual periods of $A$. Further, for some finite set $K$, both $A \cup K$ and $A \backslash K$ are periodic sets with periods $d \boldsymbol{P}$.

(ii) If $d_{1}$ is an eventual period of $A_{1}$, and if $d_{2}$ is an eventual period of $A_{2}$, then $1 \mathrm{~cm}\left(d_{1}, d_{2}\right)$ is an eventual period of $A_{1} \cup A_{2}$ and $A_{1} \cap A_{2}, \operatorname{gcd}\left(d_{1}, d_{2}\right)$ is an eventual period of $A_{1}+A_{2}$, and $d_{1}$ is an eventual period of $A_{1} \backslash K$ for any finite set $K$.

(iii) (Ascending Chain Condition) Suppose for each $i \in \boldsymbol{P}$, that $A_{i}$ is a periodic set with an eventual period d. Suppose further that for some $b \in Z$, each $A_{i}$ is bounded below by $b$. Then, for some $n \in \boldsymbol{P}, \bigcup_{i \in \boldsymbol{P}} A_{i}=\bigcup_{i=1}^{n} A_{i}$. In particular, $\bigcup_{i \in \boldsymbol{P}} A_{i}$ is a periodic set with an eventual period $d$.

We now consider sets defined by (2).

Let $X$ be a set. For $r \in \boldsymbol{P}$, we say $f$ is an r-ary operator on $X$ if $f: X^{r} \rightarrow X$. We say $f$ is a finitary operator on $X$ if $f$ is $r$-ary for some $r \in \boldsymbol{P}$, and we write $\rho(f)=r$. If $A \subseteq X$, and $f$ is a finitary operator on $X$, let $f(A)=\left\{f(a) \mid a \in A^{\rho(f)}\right\}$. If $R$ is a set of operators on $X$, let $R(A)=\bigcup_{f \in R} f(A)$. We say $A$ is closed under $f$ (under $R$ ) if $f(A) \subseteq A(R(A) \subseteq A)$.

If $A \subseteq X$, and $R$ is a set of finitary operators on $X$, let $\langle R \mid A\rangle$ be the intersection of all subsets of $X$ containing $A$ and closed under $R$. Alternatively, define a sequence $\left(A_{n} \mid n \in N\right)$, called the construction sequence of the pair $(R, A)$, inductively as follows: let $A_{n}=A \cup R\left(A_{n-1}\right)$ for $n \in \boldsymbol{P}$. It is easy to see $\langle R \mid A\rangle=\mathrm{U}_{n \in N} A_{n}$, see Theorem 2 of [3] for details, where the alternate recursion formula $A_{n}=A_{n-1} \cup R\left(A_{n-1}\right)$ is used.

We now give two fundamental theorems. The first is a special case of Theorem 9 of [3]. For the second, we only sketch a proof, as it is essentially Theorem 4 of [3].

THEOREM 1. Let $R$ be a set of operators on $\boldsymbol{Z}$ of the form $\left(x_{1}, \cdots, x_{r}\right) \rightarrow m_{1} x_{1}+\cdots+m_{r} x_{r}+c$, let $A \subseteq \boldsymbol{Z}$ let $a, b \in \boldsymbol{Z}$ then $a\langle R \mid A\rangle+b=$ $\langle S \mid a A+b\rangle$, where $S=\{g \mid g(x)=f(x)-b f(1)+(a+b-1) f(0)+b, f \in R\}$, and for $t \in Z, f(t)=f(t, t, \cdots, t)$.

Theorem 2. Let $b \in Z$, let $R$ be a set of operators on $\boldsymbol{Z}$ of the 
form $\left(x_{1}, \cdots, x_{r}\right) \rightarrow m_{1} x_{1}+\cdots+m_{r} x_{r}+c$, where $r-1, m_{1}, \cdots, m_{r} \in \boldsymbol{P}$, $c \in Z, \quad g c d\left(m_{1}, \cdots, m_{r}\right)=1$, and $\left(m_{1}+\cdots+m_{r}-1\right) b+c \in N$. Let $A \subseteq N+b$, and suppose $A$ has an eventual period $d \in P$. Then $\langle R \mid A\rangle$ is a periodic set with eventual period $d$.

Proof. Let $\left(A_{n} \mid n \in N\right)$ be the construction sequence for $(R, A)$. It is easy to show by induction on $n$, that $A_{n}$ has an eventual period $d$, and that $A_{n} \subseteq N+b$. But $\mathrm{U}_{n \in N} A_{n}=\langle R \mid A\rangle$, so the ascending chain condition gives the result.

Now to get down to business! Our first task, the most difficult, is to show that $\langle m x+n y \mid 1\rangle$ is a periodic set whenever $m, n \in \boldsymbol{P}$, $\operatorname{gcd}(m, n)=1$. Curiously, we will first consider quite a different condition, namely $m=n$.

For each $l \in N$, let $K_{l}=\left\{\left(c_{0}, \cdots, c_{h}\right) \mid h \in N, c_{0} \in\left[0,2^{l}\right]\right.$, and $c_{i} \in$ $\left[0,2 c_{i-1}\right]$ for $\left.i \in[1, h]\right\}$, and let $T_{l}=\left\{c_{0}+c_{1} m+\cdots+c_{h} m^{h} \mid\left(c_{0}, \cdots, c_{h}\right) \in\right.$ $\left.K_{l}\right\}$.

Theorem 3. Let $m \in \boldsymbol{P}$, let $S=\langle m x+m y+1 \mid 0\rangle$. Then $S=T_{0}$.

Proof. By the corollary to Theorem 3 of [3], we need only show that $T_{0}=\{0\} \cup\left(m T_{0}+m T_{0}+1\right)$. It is easy to check that $\{0\} \cup\left(m T_{0}+m T_{0}+1\right) \subseteq T_{0}$; for the reverse inclusion, let $t \in T_{0} \backslash\{0\}$. Then $t=1+c_{1} m+\cdots+c_{h} m^{h}$, where $\left(1, c_{1}, \cdots, c_{h}\right) \in K_{0}$. We need only produce $\left(d_{1}, \cdots, d_{h}\right),\left(e_{1}, \cdots, e_{h}\right) \in K_{0}$, with $d_{i}+e_{i}=c_{i}$ for each $i \in[1, h]$, for then $u=d_{1}+d_{2} m+\cdots+d_{h} m^{h-1} \in T_{0}, v=e_{1}+e_{2} m+\cdots+$ $e_{h} m^{h-1} \in T_{0}$, and hence $t=m u+m v+1 \in m T_{0}+m T_{0}+1$.

We will show, by induction on $s$, that for all $s \in[1, h]$, there exists $\left(d_{1}, \cdots, d_{s}\right), \quad\left(e_{1}, \cdots, e_{s}\right) \in K_{0}$, with $d_{i}+e_{\imath}=c_{i}$ for $i \in[1, s]$. Since $c_{1} \in\{0,1,2\}$, we can start the induction. Having found suitable $\left(d_{1}, \cdots, d_{s-1}\right)$ and $\left(e_{1}, \cdots, e_{s-1}\right)$ for $s \in[2, h]$, we need $d_{s}, e_{s} \in N$ with $d_{s}+e_{s}=b_{s}, d_{s} \leqq 2 d_{s-1}$, and $e_{s} \leqq 2 e_{s-1}$. Since $c_{s} \leqq 2 d_{s-1}+2 e_{s-1}$, such a selection of $d_{s}$ and $e_{s}$ is clearly possible, completing the induction.

TheOREM 4. Let $l, m \in \boldsymbol{P}$, with $2^{l-1} \geqq m-1$. Then $\left(2^{l} m^{l}-1\right) /(2 m-1)+m^{l} N \subseteq\langle m x+m y+1 \mid 0\rangle$.

Proof. If $\left(c_{0}, \cdots, c_{h}\right) \in K_{l}$, then $\left(1,2,4, \cdots, 2^{l-1}, c_{0}, \cdots, c_{h}\right) \in K_{0}$, thus $\left(2^{l} m^{l}-1\right) /(2 m-1)+m^{l} T_{l} \leqq T_{0}$ for all $l \in N$. But we claim $T_{l}=\boldsymbol{N}$ for $2^{l-1} \geqq m-1$; for if not, let $y$ be the smallest integer in $N \backslash T_{l}$. By hypothesis, $\left[2^{l-1}, 2^{l}\right]$ contains at least $m$ consecutive integers, thus $y=m q+r$ for some $q \in Z, r \in\left[2^{l-1}, 2^{l}\right]$. Since $(c) \in K_{l}$ for $c \in\left[0,2^{l}\right], 2^{l}<y$. Thus $q \in P$. Certainly $q<y$, thus $q \in T_{l}$ by our choice of $y$. Finally, if $b \in\left[2^{l-1}, 2^{l}\right]$ and if $\left(c_{0}, \cdots, c_{h}\right) \in K_{l}$, note 
that $\left(b, c_{0}, \cdots, c_{h}\right) \in K_{l}$; thus, $m T_{l}+\left[2^{l-1}, 2^{l}\right] \subseteq T_{l}$; hence, $y=m q+$ $r \in T_{l}$, a contradiction. Thus, no such $y$ exists, so $T_{l}=N$.

THEOREM 5. Let $l, m, n \in \boldsymbol{P}$, with $2^{l-1} \geqq m n-1$. Then $1+$ $\left((m+n)^{2}-1\right)(2 m n-1) /(2 m n-1)+\left((m+n)^{2}-1\right) m^{l} n^{l} N \subseteq\langle m x+$ $n y|1\rangle$.

Proof. $\langle m x+n y \mid 1\rangle \supseteq\left\langle m\left(m x_{1}+n y_{1}\right)+n\left(m x_{2}+n y_{2}\right) \mid 1\right\rangle=$ $\left\langle m^{2} x_{1}+m n y_{1}+m n x_{2}+n^{2} y_{2} \mid 1\right\rangle \supseteqq\left\langle m n x+m n y+m^{2}+n^{2} \mid 1\right\rangle=$ $\left((m+n)^{2}-1\right)\langle m n x+m n y+1 \mid 0\rangle+1$, by Theorem 1 . The result now follows from Theorem 4.

Corollary 1. Let $m, n \in P$, with $\operatorname{gcd}(m, n)=1$. Then, for some $a, d \in \boldsymbol{P}$ with $\operatorname{gcd}(a, d)=1$,

$$
a+d N \leqq\langle m x+n y \mid 1\rangle .
$$

Proof. Let $a=1+\left((m+n)^{2}-1\right)\left(\left(2^{l} m^{l} n^{l}-1\right) /(2 m n-1)\right)$, let $d=\left((m+n)^{2}-1\right) m^{l} n^{l}$, where $l \in P$ with $2^{l-1} \geqq m n-1$, so that (4) holds. But $\operatorname{gcd}\left(a,(m+n)^{2}-1\right)=1$, and $\operatorname{gcd}(a, m n)=\operatorname{gcd}(1+$ $\left.(m+n)^{2}-1, m n\right)=\operatorname{gcd}\left((m+n)^{2}, m n\right)=1$, since $\operatorname{gcd}(m, n)=1$.

We shall make no use of the following corollary to Theorem 5, but it is of interest in its own right. We leave the proof as an exercise.

CoRollary 2. Let $r \in \boldsymbol{P}$, let $m_{1}, \cdots, m_{r}, c \in \boldsymbol{Z}$, let $T \subseteq \boldsymbol{Z}$, with $m_{1} T+\cdots+m_{r} T+c \subseteq T$. If at least two of the $m$ 's are nonzero and if $|T| \geqq 2$, then $a+d N \cong T$ for some $a, d \in Z, d \neq 0$.

TheOREM 6. Let $m, n \in P$, with $\operatorname{gcd}(m, n)=1$. Then $T=$ $\langle m x+n y \mid 1\rangle$ is a periodic set.

Proof. By Corollary 1, $a+d \boldsymbol{N} \subseteq T$ for some $a, d \in \boldsymbol{P}$ with $\operatorname{gcd}(a, d)=1$. For each $t \in T$, let $\phi(t)$ denote the smallest element of $T$ congruent to $t$ modulo $d$. Then $k=|\phi(T)|$ is finite; and further, we may write $\phi(T)=\left\{a_{1}, \cdots, a_{k}\right\}$, where $a_{1}=1$, and for each $j \in[2, k]$, $a_{j}=m a_{j_{1}}+n a_{j_{2}}$ for some $j_{1}, j_{2} \in[1, j-1]$.

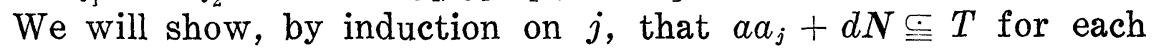
$j \in[1, k]$. Since $a_{1}=1, a a_{1}+d \boldsymbol{N} \cong T$ by hypothesis. If $j \in[2, k]$, then $a a_{j_{1}}+d \boldsymbol{N} \risingdotseq T$, and $a a_{j_{2}}+d \boldsymbol{N} \cong T$ by induction. By Lemma 5 of [3], $\quad m\left(a a_{j_{1}}+d \boldsymbol{N}\right)+n\left(a a_{j_{2}}+d \boldsymbol{N}\right) \cong T$; but $m\left(a a_{j_{1}}+d \boldsymbol{N}\right)+$ $n\left(a a_{j_{2}}+d N\right)=a a_{j}+d(m N+n N)$, completing the induction, since $m N+n N \doteq N$. 
By Theorem 5 of [3], $T$ is closed under multiplication, thus $a a_{j} \in T$ for each $j \in[1, k]$. Since $(a, d)=1$, the numbers $a a_{j}, j \in[1, k]$, are distinct modulo $d$, and thus are congruent to the number $a_{j}$, $j \in[1, k]$, in some order. Hence $a_{k}+d N \doteq a a_{k}+d N \subseteq T$ for each $k$, so $T$ has an eventual period $d$.

Corollary 3. Let $m, n \in \boldsymbol{P}$, with $\operatorname{gcd}(m, n)=1$. Let $c, t \in \boldsymbol{Z}$ with $(m+n-1) t+c \in \boldsymbol{P}$. Then $\langle m x+n y+c \mid t\rangle$ is a periodic set.

Proof. By Theorem 1,

$$
\langle m x+n y+c \mid t\rangle=\frac{(m+n-1) t+c}{m+n-1}\langle m x+n y \mid 1\rangle-\frac{c}{m+n-1} .
$$

With the grime still on our hands, we proceed to the next goal which is to extend Corollary 3 to operators $m_{1} x_{1}+\cdots+m_{r} x_{r}+c$, where $\operatorname{gcd}\left(m_{1}, \cdots, m_{r}\right)=1$. We begin with a reduction formula.

Lemma 2. Let $l, m, n \in Z$, with $l$ odd and $\operatorname{gcd}(l, m, n)=1$. Then, for some $\alpha \in P, \operatorname{gcd}\left(l, m^{\alpha}+n^{\alpha}\right)=1$.

Proof. Let $Q$ denote the finite set of primes dividing $l$, but not dividing $m n$. For each $p \in Q, m^{\alpha} \equiv n^{\beta} \equiv 1(\bmod p)$, for some $\alpha_{p}, \beta_{p} \in \boldsymbol{P} . \quad$ Let $\alpha=\operatorname{lcm}\left(\left\{\alpha_{p} \mid p \in Q\right\} \cup\left\{\beta_{p} \mid p \in Q\right\}\right)$, thus $m^{\alpha} \equiv n^{\alpha} \equiv 1$ $(\bmod p)$ for each $p \in Q$. Now we claim $g c d\left(l, m^{\alpha}+n^{\alpha}\right)=1$; if not, let $p$ divide $\operatorname{gcd}\left(l, m^{\alpha}+n^{\alpha}\right)$ for some prime $p$. Since $\operatorname{gcd}(l, m, n)=1$, $p \in Q$. But then $0 \equiv m^{\alpha}+n^{\alpha} \equiv 1+1 \equiv 2(\bmod p)$, so $p=2$, contradicting the assumption that $l$ is odd.

THEOREM 7. Let $r \in \boldsymbol{N}+2$; let $m_{1}, \cdots, m_{r} \in \boldsymbol{P}$, with $\operatorname{gcd}\left(m_{1}, \cdots, m_{r}\right)=$ 1 ; let $c \in \mathbb{Z}$, let $T \subseteq Z$ with $m_{1} T+\cdots+m_{r} T+c \subseteq T$. Then, for some $m, n \in \boldsymbol{P}$, with $\operatorname{gcd}(m, n)=1$, and for some $k \in \boldsymbol{Z}$, we have $m T+n T+k \subseteq T$.

Proof. Let $K=\left\{s \in N+2 \mid\right.$ for some $n_{1}, \cdots, n_{s} \in P$, with $\operatorname{gcd}\left(n_{1}, \cdots, n_{s}\right)=1$, and for some $\left.k \in Z, n_{1} T+\cdots+n_{s} T+k \subseteq T\right\}$. Thus $K \neq \varnothing$, since $r \in K$, and we must show $2 \in K$. Let $s=\min K$, and produce the appropriate $n_{1}, \cdots, n_{s}, k$. We can assume that $n_{1}$ is odd. If $s \geqq 3$, let $d=\operatorname{gcd}\left(n_{1}, n_{2}, n_{3}\right)$, let $n_{1}=d l, n_{2}=d m$, and $n_{3}=d n$. By Lemma 2, $\operatorname{gcd}\left(l, m^{\alpha}+n^{\alpha}\right)=1$ for some $\alpha \in P$, hence $\operatorname{gcd}\left(n_{1}, n_{2}^{\alpha}+n_{3}^{\alpha}, n_{4}, \cdots, n_{s}\right)=1$.

We now prove, by induction on $\beta$, that for all $\beta \in P$, there is a $k_{\beta} \in Z$ such that $n_{1} T+n_{2}^{\beta} T+n_{3}^{\beta} T+n_{4} T+\cdots+n_{s} T+k_{\beta} \leqq T$. This is true for $\beta=1$, with $k_{1}=k$; suppose $n_{1} T+n_{2}^{\beta} T+n_{3}^{\beta} T+$ 
$n_{4} T+\cdots+n_{s} T+k_{\beta} \subseteq T$. We can assume $T \neq \varnothing$, let $t \in T$. Then $n_{1} T+n_{2}^{\beta+1} T+n_{3}^{\beta+1} T+n_{4} T+\cdots+n_{s} T+n_{2}^{\beta}\left(\left(n_{1}+n_{3}+\cdots+n_{s}\right) t+k\right)+$ $n_{3}^{\beta}\left(\left(n_{1}+n_{2}+n_{4}+\cdots+n_{s}\right) t+k\right)+k_{\beta} \subseteq n_{1} T+n_{2}^{\beta}\left(n_{1} T+\cdots+n_{s} T+k\right)+$ $n_{3}^{\beta}\left(n_{1} T+\cdots+n_{8} T+k\right)+n_{4} T+\cdots+n_{s} T+k_{\beta} \subseteq n_{1} T+n_{2}^{\beta} T+n_{3}^{\beta} T+$ $n_{4} T+\cdots+n_{s} T+k_{\beta} \subseteq T$ by induction, thus we now only take $k_{\beta+1}=n_{2}^{\beta}\left(\left(n_{1}+n_{3}+\cdots+n_{s}\right) t+k\right)+n_{3}^{\beta}\left(\left(n_{1}+n_{2}+n_{4}+\cdots+n_{s}\right) t+k\right)+k_{\beta}$ to complete the induction.

In particular, $n_{1} T+\left(n_{2}^{\alpha}+n_{3}^{\alpha}\right) T+n_{4} T+\cdots+n_{s} T+k_{\alpha} \subseteq T$, and since $n_{2}^{\alpha}+n_{3}^{\alpha} \neq 0, s-1 \in K$, contradicting our choice of $s$. Thus $s=2$.

THEOREM 8. Let $r-1, m_{1}, \cdots, m_{r} \in \boldsymbol{P}$, with $\operatorname{gcd}\left(m_{1}, \cdots, m_{r}\right)=1$. Let $c, t \in \boldsymbol{Z}$ with $\left(m_{1}+\cdots+m_{r}-1\right) t+c \in \boldsymbol{P}$. Then $T=\left\langle m_{1} x_{1}+\cdots+\right.$ $m_{r} x_{r}+c|t\rangle$ is a periodic set.

Proof. It is easy to check that $N+t$ is closed under $m_{1} x_{1}+\cdots+m_{r} x_{r}+c$, so that $T \subseteq N+t$ and $T$ is bounded below. By Theorem 7 , for some $m, n \in \boldsymbol{P}$, with $\operatorname{gcd}(m, n)=1$, and some $k \in Z, \quad m T+n T+k \subseteq T$. Since $T \subseteq N+t,(m+n-1) t+k \in N$, but a careful examination of the proof of Theorem 7 shows in fact that $m, n$, and $k$ may be chosen so that $(m+n-1) t+k \in \boldsymbol{P}$. By Corollary 3, $S=\langle m x+n y+k \mid t\rangle$ is a periodic set; but $T=$ $\left\langle m_{1} x_{1}+\cdots+m_{r} x_{r}+c \mid S\right\rangle$, and so $T$ is a periodic set by Theorem 2 .

We are finally prepared to prove that statement (2) of Theorem 12 implies statement (1).

THEOREM 9. Let $r-1, m_{1}, \cdots, m_{r} \in \boldsymbol{P}$, with $\operatorname{gcd}\left(m_{1}, \cdots, m_{r}\right)=1$. Let $c \in Z$, let $A \subseteq Z$, with $A$ finite, and with $\left(m_{1}+\cdots+m_{r}-1\right) a+$ $c \in \boldsymbol{P}$ for all $a \in A$. Then $T=\left\langle m_{1} x_{1}+\cdots+m_{r} x_{r}+c \mid A\right\rangle$ is a periodic set.

Proof. $T=\left\langle m_{1} x_{1}+\cdots+m_{r} x_{r}+c \mid A\right\rangle=\left\langle m_{1} x_{1}+\cdots+m_{r} x_{r}+c \mid S\right\rangle$, where $S=\bigcup_{a \epsilon_{A}}\left\langle m_{1} x_{1}+\cdots+m_{r} x_{r}+c \mid a\right\rangle$. By Theorem 8, $S$ is a finite union of periodic sets, hence $S$ is a periodic set. Thus $T$ is periodic by Theorem 2 .

TheOREM 10. Let $r-1, \quad d \in \boldsymbol{P}$, let $m_{1}, \cdots, m_{r}, \quad c \in \boldsymbol{Z}$, with $\operatorname{gcd}\left(d, m_{1}, \cdots, m_{r}\right)=1$. Let $A \subseteq \boldsymbol{Z}$, and suppose that for all $a_{1}, \cdots, a_{r} \in A$, there exist $a \in A$ with $a \equiv m_{1} a_{1}+\cdots+m_{r} a_{r}+c(\bmod d)$. Then, for all $a \in A$, there exist $a_{1}, \cdots, a_{r} \in A$ with $a \equiv m_{1} a_{1}+\cdots+$ $m_{r} a_{r}+c(\bmod d)$.

Proof. For each $i \in[2, r]$, choose $k_{i}$ so large that $n_{i}=m_{i}+$ 
$k_{i} d \in \boldsymbol{P}$, and let $t=\operatorname{gcd}\left(n_{2}, \cdots, n_{r}\right) \in \boldsymbol{P}$. By the Chinese remainder theorem there is a solution $k_{1} \in \boldsymbol{Z}$ to all the congruences.

$$
k_{1} \equiv 0(\bmod p) \text { if } p \text { is a prime divising } t \text {, but not } m_{1} \text {, }
$$

and

$$
k_{1} \equiv 1(\bmod p) \text { if } p \text { is a prime dividing } g c d\left(t, m_{1}\right) \text {. }
$$

Moreover, $k_{1}$ can be chosen so large that $n_{1}=m_{1}+k_{1} d \in \boldsymbol{P}$. Note that $\operatorname{gcd}\left(n_{1}, \cdots, n_{r}\right)=1$.

Let $a \in A$, choose $k$ so large that $\left(n_{1}+\cdots+n_{r}-1\right)(a+k d)+$ $c \in \boldsymbol{P}$. Then $T=\left\langle n_{1} x_{1}+\cdots+n_{r} x_{r}+c \mid a+k d\right\rangle$ is a periodic set. Let $e$ be a period of $T$. Then $a+(k+e) d \in T$, so $a+(k+e) d=$ $n_{1} t_{1}+\cdots+n_{r} t_{r}+c$ for some $t_{1}, \cdots, t_{r} \in T$. But for each $i \in[1, r]$, $t_{\imath} \equiv a_{2}$ for some $a_{i} \in A$, and $a \equiv m_{1} a_{1}+\cdots+m_{r} a_{r}+c(\bmod d)$.

THEOREM 11. Let $r-1, m_{1}, \cdots, m_{r} \in P$, with $\operatorname{gcd}\left(m_{1}, \cdots, m_{r}\right)=1$. Let $c \in \boldsymbol{Z}$, let $T \subseteq \boldsymbol{Z}$, with $\left(m_{1}+\cdots+m_{r}-1\right) t+c \in \boldsymbol{P}$ for each $t \in T$, and assume $m_{1} T+\cdots+m_{r} T+c \subseteq T$. Then $T=\left\langle m_{1} x_{1}+\cdots+\right.$ $m_{r} x_{r}+c|A\rangle$ for some finite set $A \subseteq Z$.

Proof. Let $A=T \backslash\left(m_{1} T+\cdots+m_{r} T+c\right)$; by the corollary to Theorem 3 of [3], $T=\left\langle m_{1} x_{1}+\cdots+m_{r} x_{r}+c \mid A\right\rangle$, so we need only show $A$ is finite.

Let $d$ be an eventual period of $T$, then $d$ is also an eventual period of $m_{1} T+\cdots+m_{r} T+c$. Moreover, by Theorem 10, the residue classes modulo $d$ containing elements of $T$ are precisely the residue classes containing elements of $m_{1} T+\cdots+m_{r} T+c$, thus $T \doteq m_{1} T+\cdots+m_{r} T+c$. In particular, $A$ is finite.

Theorems 9 and 11 together prove Theorem 12, our goal. We continue these investigations in [1], where we prove the following theorem.

THEOREM. Let $r-1 \in \boldsymbol{P}$, let $m_{1}, \cdots, m_{r} \in \boldsymbol{Z} \backslash\{0\}$, with $\operatorname{gcd}\left(m_{1}, \cdots, m_{r}\right)=1$, let $c \in \mathbb{Z}$, and let $T \subseteq \boldsymbol{Z}$ with $m_{1} T+\cdots+m_{r} T+$ $c \subseteq T$. Then $T=\left\langle m_{1} x_{1}+\cdots+m_{r} x_{r}+c \mid A\right\rangle$ for some finite set $A$. Further, if $|T| \geqq 2$, either $T$ is a periodic set, or $-T$ is a periodic set, or $T$ is a finite union of residue classes modulo some $d \in \boldsymbol{P}$. Finally, $T$ is an affine transformation of a set $S \subseteq Z$, with $S+$ $\theta \subseteq S$, where $\theta=\operatorname{gcd}\left\{m_{i} m_{j} \mid i, j \in[1, r], i \neq j\right\}$.

An earlier version [4] of this paper was submitted years ago to Pacific Journal and was accepted subject to minor revision. Revision 
and strengthening of the results subsequently was carried out in connection with dissertation work [2].

\section{REFERENCES}

1. D. G. Hoffman and D. A. Klarner, Sets of integers closed under affine operatorsThe finite basis theorems, to appear.

2. D. G. Hoffman, Sets of Integers Closed under Affine Operators, Ph. D. thesis, U. of Waterloo, 1976.

3. D. A. Klarner and R. Rado, Arithmetic properties of certain recursively defined sets, Pacific J. Math., 53 (1974), 445-463.

4. D. A. Klarner, Sets Generated by Interation of a Linear Operation, Stan-CS-72-275, Computer Science Dept., Stanford University, March 1972.

Received December 13, 1976 and in revised form March 30, 1978. Research supported in part by NSF Grant No. MCS 7801908.

AUBURN UNIVERSITY

AUBURN, AL 36830

AND

SUNY-BINGHAMTON

BiNGHAMTON, NY 13901 


\title{
PACIFIC JOURNAL OF MATHEMATICS
}

\section{EDITORS}

RICHARD ARENS (Managing Editor)

University of California

Los Angeles, CA 90024

Charles W. Curtis

University of Oregon

Eugene, OR 97403

C. C. MOORE

University of California

Berkeley, CA 94720

\section{J. DUGUNDJI}

Department of Mathematics University of Southern California Los Angeles, CA 90007

R. Finn and J. Milgram

Stanford University

Stanford, CA 94305

\section{ASSOCIATE EDITORS}
E. F. BECKENBACH
B. H. NeumanN
F. WOLF
K. YOSHIDA

\section{SUPPORTING INSTITUTIONS}

\author{
UNIVERSITY OF BRITISH COLUMBIA \\ CALIFORNIA INSTITUTE OF TECHNOLOGY \\ UNIVERSITY OF CALIFORNIA \\ MONTANA STATE UNIVERSITY \\ UNIVERSITY OF NEVADA, RENO \\ NEW MEXICO STATE UNIVERSITY \\ OREGON STATE UNIVERSITY \\ UNIVERSITY OF OREGON
}

\author{
UNIVERSITY OF SOUTHERN CALIFORNIA \\ STANFORD UNIVERSITY \\ UNIVERSITY OF HAWAII \\ UNIVERSITY OF TOKYO \\ UNIVERSITY OF UTAH \\ WASHINGTON STATE UNIVERSITY \\ UNIVERSITY OF WASHINGTON
}

The Supporting Institutions listed above contribute to the cost of publication of this Journal, but they are not owners or publishers and have no responsibility for its content or policies.

Mathematical papers intended for publication in the Pacific Journal of Mathematics should be in typed form or offset-reproduced, (not dittoed), double spaced with large margins. Please do not use built up fractions in the text of the manuscript. However, you may use them in the displayed equations. Underline Greek letters in red, German in green, and script in blue. The first paragraph or two must be capable of being used separately as a synopsis of the entire paper. Items of the bibliography should not be cited there unless absolutely necessary, in which case they must be identified by author and journal, rather than by item number. Manuscripts, in triplicate, may be sent to any one of the editors. Please classify according to the scheme of Math. Reviews, Index to Vol. 39. All other communications should be addressed to the managing editor, or Elaine Barth, University of California, Los Angeles, California, 90024.

50 reprints to each author are provided free for each article, only if page charges have been substantially paid. Additional copies may be obtained at cost in multiples of 50 .

The Pacific Journal of Mathematics is issued monthly as of January 1966. Regular subscription rate: $\$ 72.00$ a year (6 Vols., 12 issues). Special rate: $\$ 36.00$ a year to individual members of supporting institutions.

Subscriptions, orders for numbers issued in the last three calendar years, and changes of address should be sent to Pacific Journal of Mathematics, P.O. Box 969, Carmel Valley, CA 93924, U.S.A. Older back numbers obtainable from Kraus Periodicals Co., Route 100, Millwood, NY 10546.

PUBLISHED BY PACIFIC JOURNAL OF MATHEMATICS, A NON-PROFIT CORPORATION

Printed at Kokusai Bunken Insatsusha (International Academic Printing Co., Ltd.). 8-8, 3-chome, Takadanobaba, Shinjuku-ku, Tokyo 160, Japan.

Copyright (C) 1978 by Pacific Journal of Mathematics Manufactured and first issued in Japan 


\section{Pacific Journal of Mathematics \\ Vol. 78, No. $2 \quad$ April, 1978}

Su-Shing Chen, Weak rigidity of compact negatively curved manifolds .... 273

Heinz Otto Cordes and D. A. Williams, An algebra of pseudodifferential operators with nonsmooth symbol ....................... 279

Herbert Paul Halpern, Normal expectations and integral decomposition of type III von Neumann algebras ......................... 291

G. Hochschild, On representing analytic groups with their

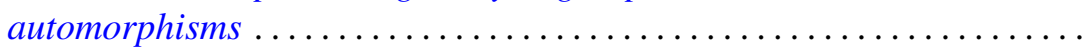

Dean G. Hoffman and David Anthony Klarner, Sets of integers closed under affine operators - the closure of finite sets ....................

Simeon Ivanov, On holomorphic relative inverses of operator-valued

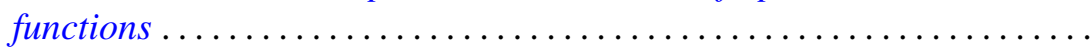

O. P. Juneja and M. L. Mogra, Radii of convexity for certain classes of

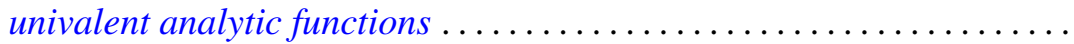

Hadi Kharaghani, The evolution of bounded linear functionals with application to invariant means.......................... 369

Jack W. Macki, A singular nonlinear boundary value problem .......... 375

A. W. Mason and Walter Wilson Stothers, Remarks on a theorem of $L$. Greenberg on the modular group ........................ 385

Kevin Mor McCrimmon, Peirce ideals in Jordan algebras . . . . . . . . . . . 397

John C. Morgan, II, On the absolute Baire property ................ 415

Gerard J. Murphy, Commutative non-Archimedean $C^{*}$-algebras ...

Masafumi Okumura, Submanifolds with L-flat normal connection of the

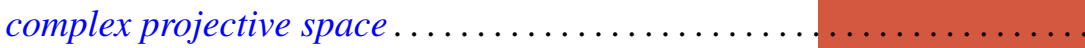

Chull Park and David Lee Skoug, Distribution estimates of barrier-crossing probabilities of the Yeh-Wiener process ...............

Irving Reiner, Invariants of integral representations ........

Phillip Schultz, The typeset and cotypeset of a rank 2 abelian group ..... 503

John Brendan Sullivan, Representations of Witt groups ....

Chia-Chi Tung, Equidistribution theory in higher dimensions . . . 